\title{
Asistencia al paciente agónico que va a fallecer en urgencias
}

\section{Care for the dying patient in emergency departments}

\author{
M.L. Iglesias ${ }^{1,3}$, A. Lafuente ${ }^{2}$
}

\section{RESUMEN}

La finalidad de este documento es aportar a los profesionales de los servicios de urgencias hospitalarios las herramientas suficientes para afrontar, según la organización y las posibilidades de cada hospital, la llegada de los pacientes en situación de últimos días. Es primordial proporcionar un entorno profesional, técnico y humano basado en conceptos, actitudes y habilidades que permitan afrontar las demandas de confort y los requerimientos emocionales y psicosociales que generan estas situaciones.

Palabras clave. Muerte. Urgencias. Paliativos. Ética. Enfermería. Agonía. Comunicación. Enfermedad terminal. Cuidadores.

\begin{abstract}
The aim of this article is to provide professionals in the hospital emergency departments with sufficient tools to face, according to the organisation and possibilities of each hospital, the admission of patients in the final days of life. It is primordial to provide a professional, technical and human environment based on concepts, attitudes and skills that make it possible to deal with the demands of comfort and the emotional and psycho-social requirements generated by these situations.
\end{abstract}

Key words. Death. Emergency care. Palliative care Ethics. Nursing. Agony. Communication. Terminal illness. Caregivers.
1. Servicio de Urgencias. Hospital de Sabadell. Corporación Parc Taulí

2. Departamento de Anestesiología y Reanimación. Clínica Universidad de Navarra. Pamplona

3. Universidad Autónoma de Barcelona

\section{Correspondencia}

M.L. Iglesias Lepine

Hospital de Sabadell. Consorcio Sanitario Parc Taulí

Apartado de Correos 196

08208 Sabadell. Barcelona

E-mail: miglesias@tauli.cat 


\section{GENERALIDADES}

Los avances en medicina y en salud pública han logrado un incremento significativo en la expectativa de vida en los países desarrollados. En España, la tasa de vejez es del $17,03 \%$ y el índice de senectud del $11,99 \%$ (porcentaje de mayores de 85 años respecto a los mayores de 65 años $)^{1}$. El aumento de la esperanza de vida, el cambio del modelo de la familia tradicional (disminución del número de miembros y la incorporación de la mujer al mundo laboral), la desaparición de la figura del médico de cabecera, la ineficiencia de las residencias geriátricas para solucionar los problemas médicos, la política sanitaria basada en el restablecimiento de la salud de los pacientes por "costes por proceso" y la restricción del número de camas hospitalarias en pro de la implementación inconclusa de los circuitos extrahospitalarios [PADES (programa de atención domiciliario de equipos de soporte), UFISS (unidad funcional interdisciplinaria sociosanitaria), hospitalización domiciliaria y camas sociosanitarios], ha provocado un incremento del número de pacientes en situación de últimos días (SUD) que acuden a los servicios de urgencias hospitalarios (SUH) para recibir asistencia y que deberían haber sido atendidos en otros niveles asistenciales $^{2-14}$.

La sociedad moderna no acepta la muerte como un hecho natural que tiene lugar en el orden biológico de las cosas, por lo que muchos pacientes fallecen en los hospitales, entornos fríos sin la privacidad y la intimidad necesarios para atender la agonía, el acompañamiento familiar y el duelo posterior ${ }^{5,7,11,15}$. En Cataluña, el $54 \%$ de los ciudadanos mueren hospitalizados $\mathrm{y}$, de este grupo, un $35 \%$ lo hace en los $\mathrm{SUH}^{16}$, espacios arquitectónicos restringidos y masificados, donde los profesionales de urgencias trabajan a un ritmo trepidante con unas plantillas limitadas y poco cuidadas (contratos basura, sueldos dispares, guardias de 24 horas, carrera profesional inexistente en algunos centros, etc.), que. junto al incremento desmesurado y progresivo de las cargas de trabajo por la dependencia de los pacientes y a la exigencia políticamente admitida de los familiares del "para eso yo pago" ${ }^{6-13}$, han acrecentado la presión de un servicio que por idiosincrasia propia lleva implícito una gran dosis de estrés, pudiendo llegar a comprometer la calidad asistencial en los períodos de colapso ${ }^{8}$.

Los planes de estudios de las universidades de la salud españolas basados en la preservación de la salud, ofrecen breves conocimientos sobre tratamientos médicos paliativos, comunicación y habilidades emocionales para cubrir la respuesta profesional, científica y humana a las múltiples necesidades que precisan los pacientes en SUD durante su fase final ${ }^{6-14}$. Los conocimientos en cuidados paliativos benefician a los pacientes terminales oncológicos así como a los pacientes con enfermedades no malignas (degenerativas del SNC, demencias muy evolucionadas, patologías pulmonares o nefrológicas en fase terminal, etc.). En la actualidad, en los SUH se instauran desiguales medidas paliativas a pacientes oncológicos y en menor proporción a pacientes con enfermedades no malignas, probablemente por la disparidad de criterios médicos entre las múltiples especialidades que integran la vida médica de un paciente, sin que exista la mayoría de las veces un responsable de todo el proceso integral ${ }^{6-14}$.

Todas las causas anteriores conducen a que "se muera mal cuando la muerte no es aceptada; se muera mal cuando los profesionales sanitarios no están formados en el manejo de las reacciones emocionales que emergen en la comunicación con los pacientes; se muera mal cuando se abandona la muerte al ámbito de lo irracional, al miedo, a la soledad, en una sociedad donde no se sabe morir" ${ }^{17}$.

La sociedad debe proponerse como objetivo del siglo XXI ayudar a los pacientes a morir en paz, con la misma categoría que la lucha contra las enfermedades por la supervivencia de la especie ${ }^{3}$. 


\section{USO CORRECTO DE LOS CONCEPTOS SOBRE EL}

FIN DE LA VIDA ${ }^{11-14,17-25}$

Utilizar los mismos términos y aclarar los significados de las palabras ayudan a los profesionales a hablar en el mismo idioma.

- Enfermedad terminal: es una enfermedad avanzada, progresiva e incurable, sin razonables posibilidades de respuesta al tratamiento, con un pronóstico de supervivencia inferior a seis meses, que se acompaña de múltiples síntomas, variables y multifactoriales.

- Enfermedad terminal oncológica: es una enfermedad terminal donde se ha comprobado que la histología del tumor no ejerce ninguna influencia en la supervivencia del paciente.

- Situación de últimos días o agonía: es el proceso que aparece en la fase final de muchas enfermedades, en la que la vida se extingue gradualmente. De duración inferior a una semana, que si se acompaña de una disminución del nivel de conciencia no suele superar los tres días, y en el cual el paciente presenta un patrón específico:

- Alteraciones físicas: aumento de la debilidad y de la postración, disminución de la ingesta con sequedad de la piel y de las mucosas, retención urinaria y fecal, variaciones de las constantes vitales con disminución de la presión arterial, pulso irregular y rápido, respiración superficial con pausas de apnea, trastornos respiratorios así como de los índices funcionales (Karnofsky, ECOG, etc.).

- Alteraciones cognitivas: desorientación, agitación o ideas delirantes.

- Alteraciones emocionales: aparición de reacciones hipoactivas como resignación o silencio y/o hiperactivas como crisis de ansiedad o de angustia.
- Confort: es la percepción subjetiva del bienestar, que requiere de una serie de medidas específicas, realistas, proporcionadas y adaptadas al ámbito de las urgencias, con especial hincapié en el control de las situaciones que producen disconfort. Para lograrlo se debe iniciar precozmente el tratamiento farmacológico de los síntomas así como prever la aparición de las diferentes complicaciones.

- Síntoma difícil: es una situación que necesita para el control clínico del síntoma la instauración de unas medidas terapéuticas más allá de las medidas habituales.

- Síntoma refractario: es una situación que no puede ser controlada, a pesar de los esfuerzos por hallar un tratamiento tolerable, sin que se comprometa el nivel de conciencia del paciente.

- Sedación en la agonía: es una decisión terapéutica prescrita sólo por facultativos, para tratar un síntoma físico o psicológico refractario a otros tratamientos.

\section{ÉTICA Y MUERTE DIGNA EN UN SERVICIO DE URGENCIAS HOSPITALARIO ${ }^{6,7,15,25-43}$}

\section{Generalidades}

El concepto de la muerte, independientemente de la idea que cada persona tenga de ella, está basado en elementos antropológicos, éticos, religiosos y jurídicos, pero para todos los seres humanos es un hecho emocionalmente impactante. Toda sociedad desarrollada debería ser capaz de enfrentarse de forma individual y social con la muerte, así como de respetar las diversas variantes que puedan derivarse de las diferentes formas de confrontarla.

La finalidad de los comités de ética hospitalarios no es educar moralmente, sino proporcionar herramientas y metodología (guías éticas hospitalarias) para tomar 
decisiones médicas prudentes y proporcionadas sobre situaciones que pueden presentar dilemas, que eviten medidas terapéuticas fútiles. El antiguo modelo paternalista que perdura en algunos médicos debe dar paso a un modelo deliberativo de respeto a la libertad y a la individualidad del paciente.

Los SUH están formados por un conjunto heterogéneo de profesionales, procedentes de distintas vivencias (ciudades, comunidades, religiones, etc.) y diferentes especialidades que marcan decisiones dispares ante la enfermedad terminal. Las guardias de presencia física están formadas por un porcentaje elevado de médicos noveles, con un deseo acentuado de adquirir experiencias en técnicas invasivas y gran heterogeneidad de reacciones lo que dificulta la toma de decisiones morales si no existe una línea tradicional de trabajo ético. Además, no todos los médicos tienen la suficiente preparación para trabajar en urgencias. Tener que tomar decisiones rápidas a pesar de soportar una gran carga de estrés -trabajar ante la mirada de todos, en turnos de trabajo rotatorios y/o guardias, con una alta presión asistencial y un número no despreciable de pacientes muy vulnerables-, poseer capacidades técnicas (conocimientos y experiencias) y habilidades en comunicación (paciente/ entorno familiar) y tener fundamentos morales basados en el respeto a la persona (libertad, intimidad y confidencialidad) y a la proporcionalidad (máximo confort, dominio de las crisis y respeto a la LET) para tomar decisiones de calidad asistencial requieren de una preparación especifica y unas cualidades humanas determinadas.

\section{Principios básicos de la bioética clínica}

Las situaciones clínicas urgentes requieren de decisiones consistentes y rápidas, por lo que la calidad de la práctica clínica no será adecuada si la técnica utilizada no va acompañada de una apropiada decisión moral sustentada en los cuatro principios básicos que gozan de consenso en la cultura occidental con diferentes matices de interpretación. El paciente, por ser autónomo, puede y debe tomar decisiones respecto a su salud. La sociedad y las instituciones deben basarse en el principio de justicia. Y el médico debe hacerlo bajo el principio de beneficencia y no maleficencia.

- Principio de no maleficencia o "primun non nocere»: evita dañar al paciente usando todos los medios disponibles de prudencia, pericia y control de calidad.

- Principio de autonomía: permite que cada persona conduzca su vida de acuerdo con sus intereses, valores, deseos y creencias. Por parte del paciente implica conocimiento, comprensión y ausencia de coacción (consentimiento libre e informado) y por parte de los profesionales información y valoración sobre la capacidad cognitiva del paciente para personalizar el plan terapéutico.

- Principio de beneficencia: procura el mayor bien del otro. No se entiende el principio de beneficencia sin el respeto a la autonomía del paciente.

- Principio de justicia distributiva: las deliberaciones morales asignan igual peso a los mismos intereses de todos los pacientes a quienes se les realizan las mismas acciones. Los médicos de urgencias, en ausencia del conocimiento necesario (información incompleta) para escoger la opción que más interesa al paciente, no pueden dejar de realizar medidas terapéuticas (abandono terapéutico) hasta que la decisión global esté argumentada tanto a nivel médico (otros miembros del mismo equipo de urgencias y/o equipo de referencia) como familiar, porque sus expectativas hacia la curación del paciente la mayoría de las veces están sobrestimadas. Al existir diferentes matices aceptables deberán encontrarse decisiones colectivas 
por consenso con otros especialistas y si es posible con especialistas en cuidados paliativos lo que garantizará la calidad asistencial.

\section{Voluntades anticipadas o testamento vital}

Tiene como objetivo básico la protección de la autonomía de la persona y es una extensión del proceso de consentimiento informado en aquellas situaciones en las que se ha perdido la capacidad de concederlo. Si existe un documento en el que el paciente ha manifestado su voluntad, se debe respetar. Si no hay documento, pero el entorno familiar conoce la voluntad del paciente, los familiares son los que decidirán. Si no existe ningún documento y el entorno familiar se inhibe, la decisión le corresponde al médico.

\section{Limitación del esfuerzo terapéutico (LET)}

Es un concepto que engloba la decisión meditada de los facultativos de no añadir más terapias e incluso retirar los tratamientos ya establecidos, al considerar que no pueden ayudar al paciente a sobrevivir o lo harían a costa de una calidad de vida insoportable. Permite la muerte en el sentido de que no la impide, pero en ningún caso la produce. En los SUH, la LET tiene características propias por la urgencia, la contextualidad, el grado de incertidumbre y el coste emocional que representa. A veces sólo se puede estimar una probabilidad aproximada de muerte, de forma incierta y basada sólo en la respuesta al tratamiento sin disponer del tiempo suficiente para preparar al paciente y al entorno familiar. Si los equipos asistenciales son multidisci- plinares, los médicos de urgencias junto a los de cuidados intensivos decidirán conjuntamente el no ingreso en las áreas de críticos anticipando la futilidad de los tratamientos altamente tecnificados. La LET es una decisión de los facultativos, por lo que debe existir una buena coordinación entre los estamentos implicados en el control de los pacientes con una enfermedad crónica avanzada eventualmente fatal y no es responsabilidad de la familia, a la que se ha de informar con tacto y consideración. En la historia clínica de los pacientes debe estar referenciado el plan de actuación con los márgenes terapéuticos que se deberían o no instaurar (fármacos vasoactivos, ventilación mecánica no invasiva, antibióticos, órdenes de no resucitación, etc.) para evitar divergencias y ofrecer una atención continuada coherente.

\section{Principio del doble efecto}

Cuando una determinada actuación tiene dos posibles efectos, uno positivo y otro negativo, si la persona actúa buscando intencionadamente el efecto positivo pero como consecuencia de ello se produce además el efecto negativo, la actuación es moralmente correcta.

\section{Órdenes de no resucitación}

La decisión por parte del médico de no iniciar maniobras de soporte vital avanzado es clínicamente apropiada y éticamente aceptable y depende del diagnóstico primario y de la evolución esperada (Tabla 1). Si el pronóstico inmediato no ofrece esperanzas, no hay ningún imperativo moral para aplicarlo y es absolutamente compatible con la continuación de los tratamientos y de las curas. 
Tabla 1. Recomendaciones sobre órdenes de no resucitación (ONR) en el Servicio de Urgencias. Comité Ético de la Corporación Parc Taulí de Sabadell (Barcelona).

\begin{tabular}{|c|c|}
\hline $\begin{array}{c}\text { Categoría } \\
\text { A }\end{array}$ & $\begin{array}{l}\text { Paciente que se realiza tratamiento y se espera dar el alta: } \\
\text { - Si PCR: se iniciarán medidas de SVA. } \\
\text { - No necesita consentimiento del paciente/representante. }\end{array}$ \\
\hline $\begin{array}{c}\text { Categoría } \\
\text { B }\end{array}$ & $\begin{array}{l}\text { Paciente que se desconoce el pronóstico de la enfermedad subyacente: } \\
\text { - Si PCR: se iniciarán medidas de SVA hasta que se evidencie su fracaso o se obtenga la } \\
\text { información de que pertenece a la categoría C. }\end{array}$ \\
\hline $\begin{array}{l}\text { Categoría } \\
\text { C }\end{array}$ & $\begin{array}{l}\text { Paciente en SUD por la historia clínica: } \\
\text { - Si PCR y existen órdenes de no resucitar: no hay imperativo moral de instaurar un } \\
\text { tratamiento que no se espere que mejore las condiciones del paciente. } \\
\text { - No requiere permiso del paciente/representante pero sí información. }\end{array}$ \\
\hline $\begin{array}{c}\text { Categoría } \\
\text { D }\end{array}$ & $\begin{array}{l}\text { Paciente con enfermedad crónica y debilitante (física y/o mental) relativamente aguda y } \\
\text { amenazadora para la vida, pero la muerte no parece inminente: } \\
\text { - Si PCR y en la historia clínica no está claro el beneficio del SVA, optar por la preferencia } \\
\text { paciente/representante. Si hay dudas, buscar quórum con el equipo de guardia y } \\
\text { posteriormente retirar el SVA si se cree oportuno. }\end{array}$ \\
\hline $\begin{array}{l}\text { ¿Pueden } \\
\text { cambiarse } \\
\text { las ONR? }\end{array}$ & $\begin{array}{l}\text { - Si la situación clínica y el pronóstico cambia: puede convertirse en una categoría A. } \\
\text { - Si el paciente/representante de la categoría D quiere rescindirla. } \\
\text { - Si al paciente/representante de la categoría D se le tuviera que realizar un procedimiento } \\
\text { primordialmente quirúrgico, se podría considerar la rescisión temporal de las ONR en } \\
\text { cuanto a los riesgos específicos de la intervención. }\end{array}$ \\
\hline
\end{tabular}

Abreviaturas. PCR: parada cardiorrespiratoria; SUD: situación de últimos días; SVA: soporte vital avanzado.

\section{Intervención fútil}

Es cualquier acción diagnóstica o terapéutica que no beneficie al paciente. El profesional tras una evaluación exhaustiva de los datos clínicos de los que dispone no tiene ninguna obligación ética de iniciarla y, si ya se ha iniciado, debería proceder a retirarla.

\section{Obstinación terapéutica}

Es la instauración o continuación de medidas médicas carentes de ningún otro sentido que el de prolongar la vida del paciente cuando éste está abocado a una muerte irreversible. Pretender prolongar a toda costa la vida humana meramente biológica es un grave atentando a la dignidad de la persona. No todo lo técnicamente posible es éticamente admisible, porque la desproporcionalidad injustificada, más allá de lo que es médicamente razonable, sólo prolonga la agonía.

\section{Distanasia}

Es el inicio o continuación de un tratamiento terapéutico desproporcionado que prolonga la agonía de enfermos desahuciados.

\section{Eutanasia}

Es la muerte del paciente en «situación de capacidad" por petición expresa de él mismo en un tiempo estipulado, que se produce de forma directa mediante una relación causa-efecto única e inmediata, en un contexto de sufrimiento entendido como "dolor total» por una enfermedad incurable que el paciente experimenta como inaceptable y que no ha podido ser mitigada por otros medios. Son realizadas por profesionales sanitarios (estados de Holanda, Bélgica y Luxemburgo) que conocen a los pacientes y mantienen con ellos una relación clínica significativa. 


\section{Suicidio médicamente asistido}

Es cuando la actuación del profesional sanitario (estado de Oregón) se limita a proporcionar al paciente los medios imprescindibles para que sea él mismo quien se produzca la muerte.

\section{PERFIL DEL PACIENTE ${ }^{7-14}$}

Los pacientes con una enfermedad crónica avanzada eventualmente fatal son los que fallecen habitualmente en los SUH. No sólo se produce porque el paciente se encuentra en SUD (ej.: cáncer o nefropatía terminal) sino por una crisis en una enfermedad con limitación progresiva (ej.: enfermedades cardiopulmonares) o por una complicación en un paciente con un deterioro progresivo y lento (ej.: anciano frágil con demencia evolucionada). El médico como profesional debe tener la solidez técnica y científica que le permita arbitrar correctamente ante la vida y la muerte. La opción ponderada tiene como finalidad la moderación terapéutica y el control del síntoma difícil y/o refractario.

\section{COMUNICACIÓN ENTRE EL PERSONAL SANITARIO Y EL BINOMIO PACIENTE- ENTORNO FAMILIAR ${ }^{44-52}$}

\section{La comunicación}

Es una herramienta básica para el ejercicio del trabajo habitual de los profesionales de urgencias que ayuda a comprender a los pacientes y a los familiares como personas. El factor más importante que determina el grado de satisfacción de los usuarios siempre está relacionado con el trato y la información. Comunicar que un paciente está en SUD o ha fallecido en la sociedad actual es informar de una mala noticia, y más si se realiza en un marco tan técnico como el SUH. Los profesionales de urgencias deben de aprender a admitir la muerte, tanto de forma individual como social, y manejar habilidades comunicativas para poder notificarla al entorno familiar.

\section{Información de malas noticias al entorno familiar}

Manejar las malas noticias disminuye el impacto emocional y tener poca habilidad genera un sufrimiento añadido. El lenguaje verbal conciso y asequible, sin detalles innecesarios ni mensajes exagerados, en el idioma habitual del interlocutor, con un tono pausado, es necesario para que la información sea creíble. De forma progresiva, pero constante y con calma, se repetirá el mismo mensaje tantas veces como sea necesario, utilizando frases cortas con un vocabulario neutro sin abusar de los tecnicismos médicos y, sobre todo, sabiendo escuchar. El adjunto, o el residente de los últimos años, deberá ir acompañado por otro miembro del equipo asistencial y, si es posible, de un médico residente de primeros años para su formación, presentarse (nombre y apellido) y dirigirse a todos los miembros de la familia como un grupo. Antes de proceder a dar la información deben haberse revisado los objetivos del plan terapéutico y, posteriormente, se sentarán en el despacho de información (predisposición de dedicar tiempo a la familia), frente a los familiares (aumenta el grado de implicación), al mismo nivel visual (disposición a comunicarse), inclinándose y aproximándose a los familiares (transmite implicación), utilizando una postura relajada (sin cruzar los brazos y las piernas) y una estética cuidada (un pijama con manchas genera desconfianza), mostrando afecto y formalidad (propician un clima que facilita la escucha y la empatía) y seguridad (todas las opiniones expresadas deben estar fundamentadas).

A pesar de que las familias hayan sido informadas de las limitaciones terapéuticas, cuando llegan al SUH pueden negar la realidad o hacer peticiones de eutanasia lo que provoca una situación de gran impacto emocional, por lo que debe asegurarse que la información (ética, deontológica y legal) sobre la LET llegue a la mayor parte del entorno familiar. Deben especificarse los síntomas que puedan aparecer y las probables complicaciones del control de los mismos. Se debe aconsejar que sean 
prudentes en los comentarios delante del paciente, aunque se encuentre en coma, y que mantengan la comunicación y el contacto físico, implicándolos en el cuidado y en el alivio de los síntomas. Todos los profesionales deben saber controlar situaciones difíciles como son los sentimientos de culpa de algunas familias (posturas de alejamiento o conductas inapropiadas propias de la inmadurez personal o de los hábitos culturales) mediante estrategias psicológicas de confrontación y de control de las emociones e intolerantes con las conductas agresivas (llamar a los guardias de seguridad para protección personal).

Los errores más frecuentes que se producen habitualmente en la comunicación con el entorno familiar son no comprobar la capacidad de compresión de los interlocutores y creer que lo han entendido a la primera explicación, adelantarse a las preguntas, dar más información de la que han pedido y expresarse con imprecisión intentando decir muchas ideas en un único enunciado.

\section{Información de malas noticias a los pacientes}

Los pacientes en SUD se esfuerzan por conservar la esperanza ante una situación médica cada vez más difícil y una parte de ellos no desea conocer la verdad. Hay que averiguar lo que saben o intuyen y escuchar con atención sus necesidades, procurando causar el menor impacto posible, por lo que sólo se informará de la verdad soportable. Se ofrecerá apoyo verbal y no verbal (respetando los silencios), sin adoptar actitudes de negación de la gravedad. Si el paciente está confuso o somnoliento, se deben utilizar frases cortas y preguntas sencillas sobre si tiene dolor, se encuentra cómodo o necesita algo, y se evitará cualquier signo de prisa.

\section{Información y comunicación al paciente $y$ al entorno familiar}

Las enfermeras, las auxiliares de enfermería y los camilleros deben mantener has- ta el final el contacto humano-emocional con el paciente y optimizar la satisfacción de sus necesidades. Si está confuso o somnoliento, deben comunicarse con él mediante frases cortas y preguntas sencillas (¿tiene dolor? ¿está cómodo? etc.). Se debe evitar la sensación de prisa, sin adoptar actitudes de negación y mostrando siempre preocupación por el confort del paciente. Es posible comunicarse sin palabras, a veces una mirada basta para transmitir un mensaje. No se debe ser demasiado estricto con las normas y protocolos sino que hay que adaptarlos a las necesidades de cada paciente, porque el paciente necesita atención, benevolencia, simpatía, calor humano y compañía, y esto requiere habilidad, delicadeza, tacto y discreción.

\section{LOGÍSTICA $^{4,6-7,10-11,14,18,39,47,49,51-55}$}

La comunicación interdisciplinaria entre los niveles asistenciales y la adecuada información por el especialista y/o el médico de atención primaria sobre el proceso de evolución del paciente deberían evitar la llegada de la mayoría de los pacientes en SUD a urgencias cuando presienten la muerte. Pero una proporción no desdeñable de estos pacientes acude por claudicación familiar al no poder ser asumidos desde el domicilio por múltiples factores del proceso clínico o del entorno social. La familia conoce el estado de gravedad del paciente pero no lo asume o no ha encontrado un recurso material o un apoyo psicológico para asumir los cuidados y las manifestaciones de los síntomas del paciente. Los SUH son un dispositivo más de los hospitales, que deben estar preparados para atender a este tipo de pacientes como un eslabón de la misma cadena.

\section{Miembros que forman parte del equipo de intervención}

- Equipo asistencial: se necesita un equipo de cuidados mínimos formado por un médico, una enfermera y una auxiliar de enfermería, al que se incorporará cuando sea factible el facultativo/s del equipo de referencia. 
- Equipo de soporte:

- Interlocutora o intermediaria: es una de las figuras que ha incrementado las plantillas de los SUH en los últimos años y que ha mejorado de forma substancial el grado de comunicación entre el entorno familiar y los sanitarios al servir de enlace.

- Unidad de cuidados paliativos (UFISS, PADES): la comunicación directa con los equipos de soporte domiciliario y hospitalario permite realizar consultas puntuales sobre la valoración de pacientes complejos, así como la gestión y reorientación hacia otros dispositivos.

- Soporte religioso: el equipo asistencial debe favorecer el contacto del paciente con los diferentes representantes religiosos, porque se ha demostrado que la creencia en el más allá disminuye la angustia y la desesperanza.

- Administrativo/s: para salvaguardar la intimidad y la confidencialidad del paciente en SUD, los administrativos responsables de la información sobre la ubicación de los pacientes no deben de dar ningún tipo de información sin consultar con el equipo asistencial y/o la interlocutora o intermediaria.

- Otros equipos de apoyo que favorecen la excelencia: psicóloga, trabajadora social, etc.

\section{Documentación}

- Sistema informático de admisiones: se recomienda la existencia de un apartado en la ficha técnica que recoja si existen disposiciones éticas (documento de voluntades anticipadas), porque una de las principales trabas con la que se enfrenta la sociedad para consolidar los derechos de los pacientes es el desconocimiento de los mismos por parte de los médicos y a veces del entorno familiar.
- Informe clínico: la historia clínica con los criterios terapéuticos y el plan de control de síntomas establecido por el equipo de referencia (equipo de atención primaria, PADES o UFISS) evitan los riesgos de la pérdida de información y la dilución de la responsabilidad. Para asegurar la misma línea de actuación entre los diferentes turnos, en las hojas de curso clínico se debe anotar cualquier decisión y/o acción tomada. Si el paciente retornara al domicilio, se entregará a los familiares el informe de asistencia con los cambios y las intervenciones realizadas para la continuidad del plan terapéutico por el equipo de referencia.

\section{Infraestructura arquitectónica}

Cuando la muerte se produce en el SUH, la estructura arquitectónica y organizativa no favorece el tratamiento del paciente en SUD. Para garantizar la máxima intimidad y comodidad, los SUH han de adaptarse a la realidad de la demanda, buscando estrategias de adecuación de los espacios arquitectónicos.

- Box individual: se ha de evitar a toda costa que la muerte acontezca en una camilla o en un box compartido o en un pasillo, hecho no inhabitual como lo demuestra el trabajo de Iglesias ML y $\operatorname{col}^{7}$ con una muestra de 145 pacientes fallecidos, donde el $62,1 \%$ de los fallecidos en urgencias fueron ubicados en una camilla en un box individual y el $15,9 \%$ en una cama en una sala compartida, por lo que es imprescindible que se disponga de boxes individuales con camas o camillas con colchonetas adecuadas para el confort, lo más alejados posibles de la primera línea de actuación urgente, que permitan la intimidad y el acompañamiento familiar. El número de familiares debe de adecuarse a las dimensiones de cada SUH, con un horario de visitas flexible que permita el respeto al propio paciente y al resto de los usuarios que se encuentran en urgencias. 
- Despacho de información: la información se debe dar en un espacio físico apropiado y evitar así pasillos y boxes compartidos porque es el mínimo respeto que se requiere para salvaguardar la intimidad del paciente. Se recomienda que sea un lugar acogedor, que disponga de una mesa redonda con sillas suficientes, que esté exenta de ordenadores y utensilios médicos y que disponga de una alarma conectada al servicio de seguridad del hospital para preservar a los profesionales de las reacciones agresivas y violentas.

\section{PLAN DE ACTUACIÓN \\ PARA EL CONTROL DE LOS SÍNTOMAS ${ }^{18-34,40-41,46,56-61}$}

La primera actuación que se debe llevar a cabo cuando el paciente llega al SUH es transmitir tranquilidad y seguridad para afianzar el grado de confianza. Si el paciente está somnoliento, valorar el lenguaje no verbal para detectar el dolor o la agitación y, si está consciente, él mismo marcará sus propias necesidades. La segunda actuación es ajustar el tratamiento farmacológico. Para ello se deben utilizar fármacos de eficacia demostrada a dosis adecuadas, con pautas sencillas y fijas que controlen los síntomas que produzcan disconfort. Y tercero, hay que valorar escrupulosamente la idoneidad de la realización de pruebas diagnósticas, sobre todo si exigen desplazamientos y movilizaciones porque son penosas para el paciente. Los profesionales de urgencias deben comprender que el éxito en el tratamiento del paciente no siempre es mantenerlo con vida a toda costa, sino que en ocasiones la mejor asistencia es brindarle la posibilidad de morir con dignidad con una asistencia paliativa de calidad respetuosa con sus valores y sus creencias.

\section{RECOMENDACIONES}

\section{NO ADMINISTRAR SUEROTERAPIA}

Es incoherente administrar una pauta de fluidoterapia como prueba de que se está haciendo todo lo posible, porque la hidratación del paciente no contribuye al control de los síntomas y puede empeorar las secreciones respiratorias, los edemas, los vómitos y obligar al sondaje urinario en caso de retención de orina. Por ello, se deben pautar cuidados locales que han demostrado ser mucho más eficaces para disminuir la sed.

\section{MEDIDAS FARMACOLÓGICAS DEL CONTROL DEL CONFORT}

\section{Elegir la vía de administración}

- Vía subcutánea: los pacientes en SUD presentan una disminución importante de la masa muscular secundaria al síndrome de consunción y, en el caso de los pacientes oncológicos, las venas pueden estar esclerosadas por la quimioterapia, por lo que se recomienda la administración de fármacos por vía subcutánea (SC) en bolo o mediante una bomba de infusión continua (IC), al ser poco iatrogénicos y bien tolerados (excepto los fármacos liposolubles y las grandes perfusiones). La vía SC es fácil de utilizar, con pocos efectos secundarios y complicaciones y no limita el movimiento del paciente.

- Vía intravenosa (IV): es útil sólo si existe anasarca, riesgo de hemorragia, rechazo a otras vías por parte del paciente o la necesidad de usar fármacos de uso exclusivo por esta vía.

- Vía espinal o transdérmica: se debe de mantener si alguno de los síntomas está controlado por medio de esta vía.

\section{Fármacos}

No se deben pautar los medicamentos que el paciente lleva tomando toda la vida si no tienen eficacia inmediata sobre el confort. En el caso de la insulina, los antidiabéticos orales, los antiarrítmicos, las heparinas, los diuréticos, los antibióticos, 
los antidepresivos, los laxantes, los antihipertensivos, los broncodilatadores (aerosoles), los anticomiciales, etc.

\section{Dolor}

Es clínica y éticamente inaceptable usar placebos para controlar el dolor porque morir con dignidad significa morir sin dolor. Se deben utilizar fármacos de tercer escalón como la morfina. Es mejor conocer pocos fármacos que tener un conocimiento superficial de todos los disponibles. No debe olvidarse que los opioides:

- No tienen "efecto techo".

- No se pueden prescribir a demanda sino a intervalos fijos para conseguir su máxima efectividad.

- La dosis adecuada para combatir el dolor es la dosis mínima requerida para obtener el efecto farmacológico deseado y que se puede incrementar casi indefinidamente.

- No se deben administrar varios opiáceos a la vez para controlar el dolor refractario porque pueden interaccionar entre sí disminuyendo su efectividad.

- En los pacientes que llevan tratamiento previo y se presenta una crisis de dolor agudo se debe incrementar en un $33 \%$ de su dosis habitual ( $1 / 6$ de la dosis de morfina/24 h o el equivalente a la mitad de la dosis del parche de fentanilo en forma SC-IV) y posteriormente normalizar la nueva pauta sumando la dosis basal más las dosis de rescate.

- En los pacientes sin tratamiento previo con opioides, se recomienda empezar con dosis bajas de cloruro mórfico (5-10 mg/4 h SC-IV), que serán reguladas individualmente (cada 30 minutos) hasta obtener el efecto deseado. Se ha demostrado en varios estudios con gran número de pacientes y una metodología correcta que el tratamiento del dolor intenso con analgésicos opioides a dosis estándar $(0,1 \mathrm{mg} / \mathrm{kg})$ no cumple criterios terapéuticos, como lo demuestra el trabajo de Aubrun y col $^{58}$ con una muestra de 4.525 pacientes, donde los requerimientos de opioides para paliar el dolor fueron de $0,173 \mathrm{mg} / \mathrm{kg}$ en hombres y de $0,192 \mathrm{mg} / \mathrm{kg}$ en mujeres hasta los 75 años de edad, en donde se igualan las diferencias. Por ello se debe formalizar un ajuste terapéutico progresivo y acorde a las necesidades analgésicas del paciente hasta alcanzar el confort.

\section{Náuseas y vómitos}

Se deben descartar los antagonistas 5-HT3, las ortopramidas y los antihistamínicos y sustituirlos por neurolépticos:

- Haloperidol: 2,5-10 mg/24 h en IC/SC o IC/IV o en bolo cada 6-8 h SC.

- Levomepromazina: 50-150 mg/24 h IC/SC o IC/IV o en bolo 6-8 h SC.

- La dexametasona es el fármaco de elección para controlar las náuseas y vómitos por hipertensión endocraneal porque está demostrada su efectividad en el confort.

\section{Mioclonías y convulsiones}

Se deben sustituir los anticomiciales por benzodiacepinas en pacientes con antecedentes previos o que presentan lesiones ocupantes de espacio para evitar la aparición de las crisis, sin olvidar que la morfina puede provocar mioclonías como signo de neurotoxicidad:

- Midazolam: bolo de 5-10 mg seguido de $15-30 \mathrm{mg} / 24 \mathrm{~h} \mathrm{IC/SC}$ o IC/IV

- Clonazepam: bolo $1 \mathrm{mg}$ seguido de 1,5-3 mg/24 h IC/SC o IC/IV.

\section{Síndrome confusional agudo (agitación psicomotriz y delirium hiperactivo)}

Antes de iniciar cualquier pauta farmacológica hay que descartar procesos intercurrentes como el dolor, la presencia de globo vesical o la impactación fecal: 
- Haloperidol (potente efecto antipsicótico y escaso efecto sedante): $2 \mathrm{mg}$ iniciales seguido de $1 \mathrm{mg}$ cada $20-30 \mathrm{~min}$ hasta controlar el síntoma, seguido de una pauta de 1,5-2,5 mg/8 h SC o IV.

- Midazolam (sin efecto antipsicótico pero potente efecto sedante): se debe utilizar cuando el síntoma no está controlado después de la administración de tres dosis de haloperidol: $5 \mathrm{mg}$ cada $5 \mathrm{~min}$ SC hasta que ceda el síntoma y posteriormente administrar una infusión de 30-120 $\mathrm{mg} / 24 \mathrm{~h} \mathrm{IC/SC}$ o IC/IV.

- Si a pesar de los fármacos utilizados el síntoma es refractario, considerar la sedación.

\section{Disnea}

Es un síntoma de difícil control que genera una gran ansiedad al paciente y al entorno familiar.

- Morfina (cuando el paciente usa la musculatura respiratoria accesoria): $2,5 \mathrm{mg} / 4 \mathrm{~h}$ SC o IV.

- Midazolam (cuando el paciente usa la musculatura abdominal): 5-10 mg SC o IV.

- Oxigenoterapia a demanda (si hipoxemia o ansiedad) por medio de gafas nasales. Evitar las mascarillas porque producen sensación de ahogo

- Furosemida (cuando exista insuficiencia cardiaca refractaria en situación terminal porque mejora la disnea): 20-40 mg SC o en IC/SC.

- Si a pesar de los fármacos utilizados el síntoma es refractario, considerar la sedación.

\section{Estertores y respiración ruidosa}

Aparece en la mayoría de los pacientes agónicos y produce una gran angustia en la familia. Se recomienda utilizar anticolinérgicos y no retirarlos a pesar de la mejoría o desaparición de los síntomas:

- Escopolamina: 0,5-1 mg/4-6 h SC o IV o en IC/SC o IC/IV. Añadir midazolan:
10-15 mg/24 h para prevenir la agitación que se asocia con el uso de la escopolamina.

- Butilbromuro de hioscina: $20 \mathrm{mg} / 4$ 6h SC o IV.

\section{Fiebre}

Sólo se debe tratar en los casos que produce malestar. Se recomienda retirar o aligerar la ropa de abrigo, no poner compresas frías porque producen disconfort y utilizar antitérmicos antinflamatorios no esteroideos si es preciso:

- Ketorolaco: $30 \mathrm{mg} \mathrm{SC} \mathrm{o} \mathrm{IV}$

- Metamizol: 2 g IV

- Paracetamol: 1g IV

\section{Hemorragia}

Es una situación de difícil manejo, por ser especialmente angustiosa para el paciente y su familia, y la muerte puede llegar en pocos minutos. Por ello se deben tener preparadas toallas de color oscuro y una jeringa precargada de $15 \mathrm{mg}$ de midazolam que se administrará cada 5 min SC o IV hasta conseguir la sedación profunda.

\section{Oclusión intestinal}

- Levomepromazina: 50-150 mg/24 h IC/SC o IC/IV o en bolo 6-8 $\mathrm{h} \mathrm{SC}$.

- Escopolamina: 0,5-1 mg/4-6 h SC o 3-6 mg IC/SC. Añadir midazolan (10-15 $\mathrm{mg} / 24 \mathrm{~h}$ ) para prevenir la agitación que se asocia con el uso de la escopolamina.

- Butilbromuro de hioscina: $120 \mathrm{mg} / 24 \mathrm{~h}$ IC/SC o IC/IV o en bolo $20 \mathrm{mg} / 6-8 \mathrm{~h}$ SC.

\section{MEDIDAS NO FARMACOLÓGICAS PARA EL CONTROL DEL CONFORT}

Es de vital importancia respetar la imagen corporal y la intimidad del paciente.

\section{Cambios posturales}

Realizarlos con suavidad, utilizando almohadas para obtener posturas antiálgicas 
y prevenir el eritema por presión, colocándolas entre las piernas, los codos, etc. La posición más cómoda para el paciente es decúbito lateral con piernas flexionadas.

\section{Cuidados de la piel}

Realizar la higiene y el cambio de sábanas con suavidad si el paciente está estable. Tras el aseo, secar bien la piel, sobre todo en las zonas de pliegues y dar masajes con cremas hidratantes incluyendo a la familia en su cuidado. Si existieran úlceras, el objetivo es evitar el dolor mediante la aplicación tópica de anestésicos locales y cambios posturales cada 4-6 h. El mal olor se tratará mediante gel de metronidazol al $2 \%$ y apósitos de carbón activo y el sangrado mediante adrenalina tópica cada $6 \mathrm{~h}$ y compresión con gasas.

\section{Cuidados de la boca}

Las intervenciones sobre la cavidad oral contribuyen en una gran medida al confort del paciente, por lo que se debe retirar la dentadura postiza si no está adaptada para prevenir desplazamientos. Si está bien anclada, se debe limpiar mediante torunda o cepillo dental infantil incluyendo a la familia en el cuidado. Si hay sarro, humedecer la torunda con peróxido de hidrógeno diluido (agua oxigenada). Si existen erosiones en la cavidad bucal, dar toques con lidocaína al $2 \%$ o topicaína en vaporizador. Hidratar con frecuencia la boca con agua (cucharita, jeringa o gasas) porque el reflejo de succión se conserva hasta el final de la vida, y la sequedad de los labios y de la mucosa oral producen dolor. No usar colutorios que contengan alcohol porque la resecan, ni vaselina porque al ser hidrófoba producirá más sequedad si el paciente está deshidratado.

\section{Control de la disnea}

Evitar las prendas constrictivas, elevar la cabeza del paciente con el cuello ligeramente flexionado y permeabilizar las vías aéreas limpiando las fosas nasales con suero salino. Si está consciente, la posición de
Fowler favorece la ventilación pulmonar, y si está inconsciente la semipronación evita la aspiración. Si existe derrame pleural, colocar al paciente en decúbito lateral sobre el lado afectado. Si existen secreciones no se deben aspirar porque sólo se consigue un efecto de corta duración a expensas de un gran malestar, por lo que no es una técnica recomendable.

\section{Control del estreñimiento}

No utilizar ni enemas ni laxantes, porque en esta fase del proceso no suele incomodar al paciente.

\section{Control de la incontinencia urinaria o de la retención aguda de orina}

Si el paciente está agitado comprobar si es la causa del síntoma. Si la frecuencia del cambio de empapadores o la presencia de un globo vesical produce dolor, a pesar de la inminencia de la muerte, proceder al sondaje vesical.

\section{Control del delirium}

Trastorno neurológico orgánico, de comienzo agudo, con cambios en la atención y la percepción, donde es fundamental el papel de la enfermería y la familia en la reorientación del paciente en tiempo, espacio y persona, para evitar la confrontación. En ningún caso se recomienda la contención.

\section{SEDACIÓN EN LA AGONÍA}

Es una decisión terapéutica prescrita sólo por facultativos para tratar un síntoma físico (delirium, disnea, dolor y hemorragia) o psicológico (crisis de angustia o pánico) refractario a otros tratamientos. El objetivo final es aliviar adecuadamente uno o más síntomas pertinaces que le causan sufrimiento, aunque aparezca como efecto secundario la disminución profunda y previsiblemente irreversible del nivel de conciencia ("principio del doble efecto") ${ }^{18}$, a diferencia del coma farmacológico o la eutanasia, cuyo objetivo final es la muerte 
del paciente mediante la administración de un fármaco letal. Para indicar una sedación deben cumplirse cuatro requisitos:

- Uno o más síntomas refractarios a tratamientos previos.

- Respetar los deseos expresados por el paciente mediante el consentimiento informado y, si no es factible, por el de su familia o representante.

- Consenso con el equipo asistencial y el entorno familiar con la presencia de al menos dos profesionales y dos familiares.

- Debe estar escrito y meditado en la historia clínica del paciente.

\section{Preparación antes de iniciar la sedación}

- Utilizar la escala de Ramsay para disminuir el nivel de conciencia, por su simplicidad de uso y fiabilidad (Tabla 2).

- Elegir la vía de administración.

- Mantener los analgésicos.

- Simplificar el tratamiento.

- Calcular la dosificación del fármaco a utilizar (Tablas 3 y 4 ).

Tabla 2. Escala de RAMSAY.

\begin{tabular}{|c|l|}
\hline NIVEL & \\
\hline $\mathbf{1}$ & Agitado y ansioso \\
\hline $\mathbf{2}$ & Tranquilo y colaborador \\
\hline $\mathbf{3}$ & $\begin{array}{l}\text { Despierta bruscamente con estímulo } \\
\text { verbal o percusión glabelar }\end{array}$ \\
\hline $\mathbf{4}$ & Respuesta perezosa a estímulo glabelar \\
\hline $\mathbf{5}$ & Respuesta a estímulos dolorosos \\
\hline $\mathbf{6}$ & No respuesta \\
\hline
\end{tabular}

Tabla 3. Algoritmo de actuación de la sedación en la agonía.

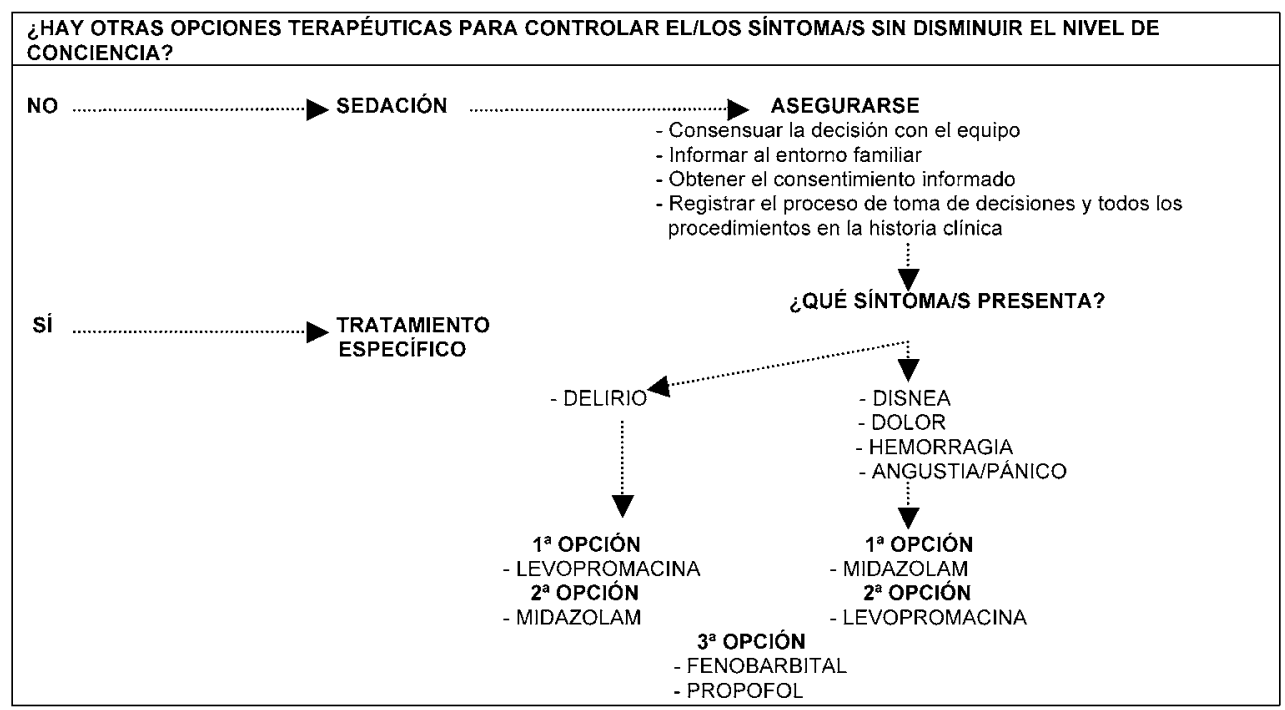


Tabla 4. Dosis farmacológicas para la sedación.

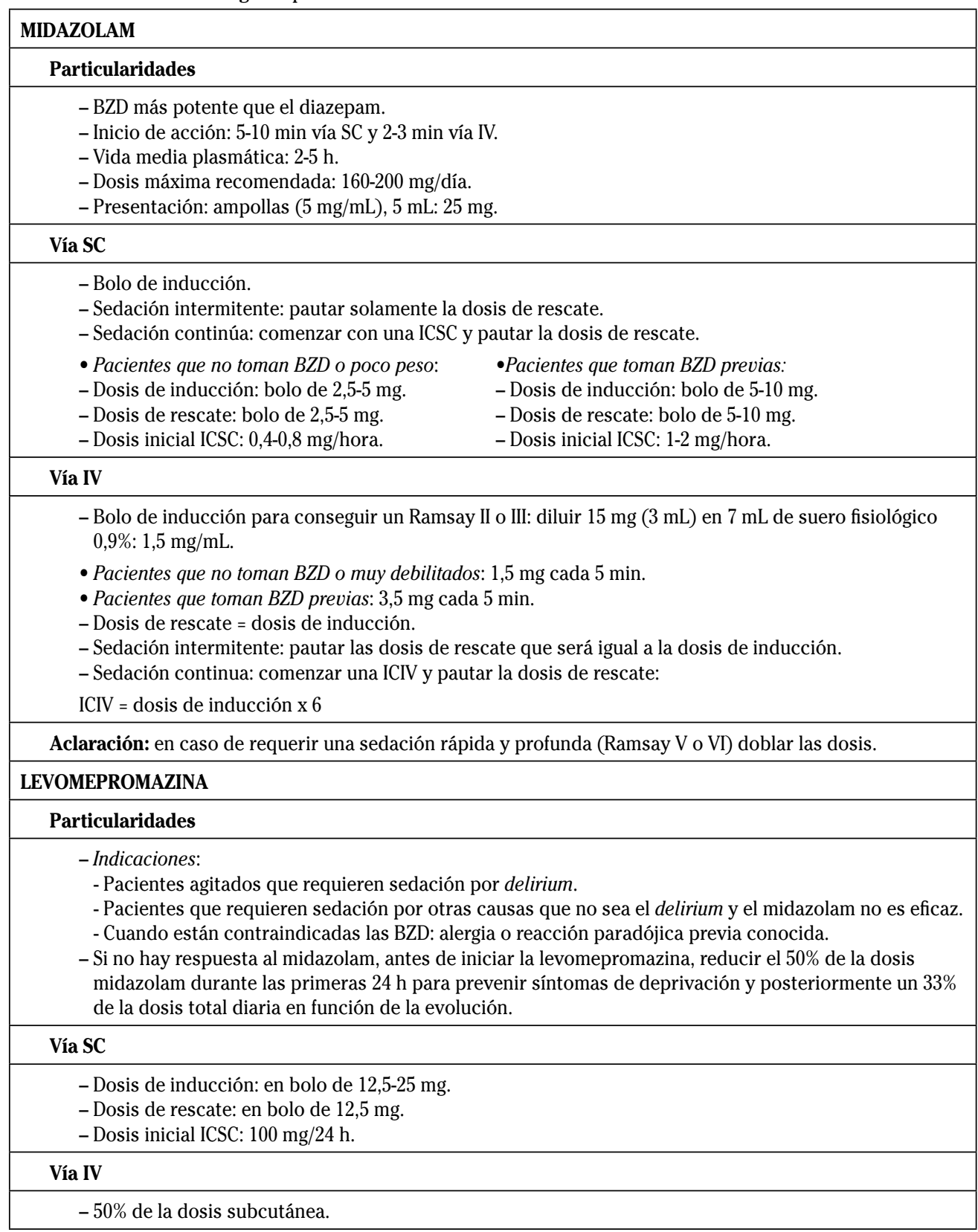




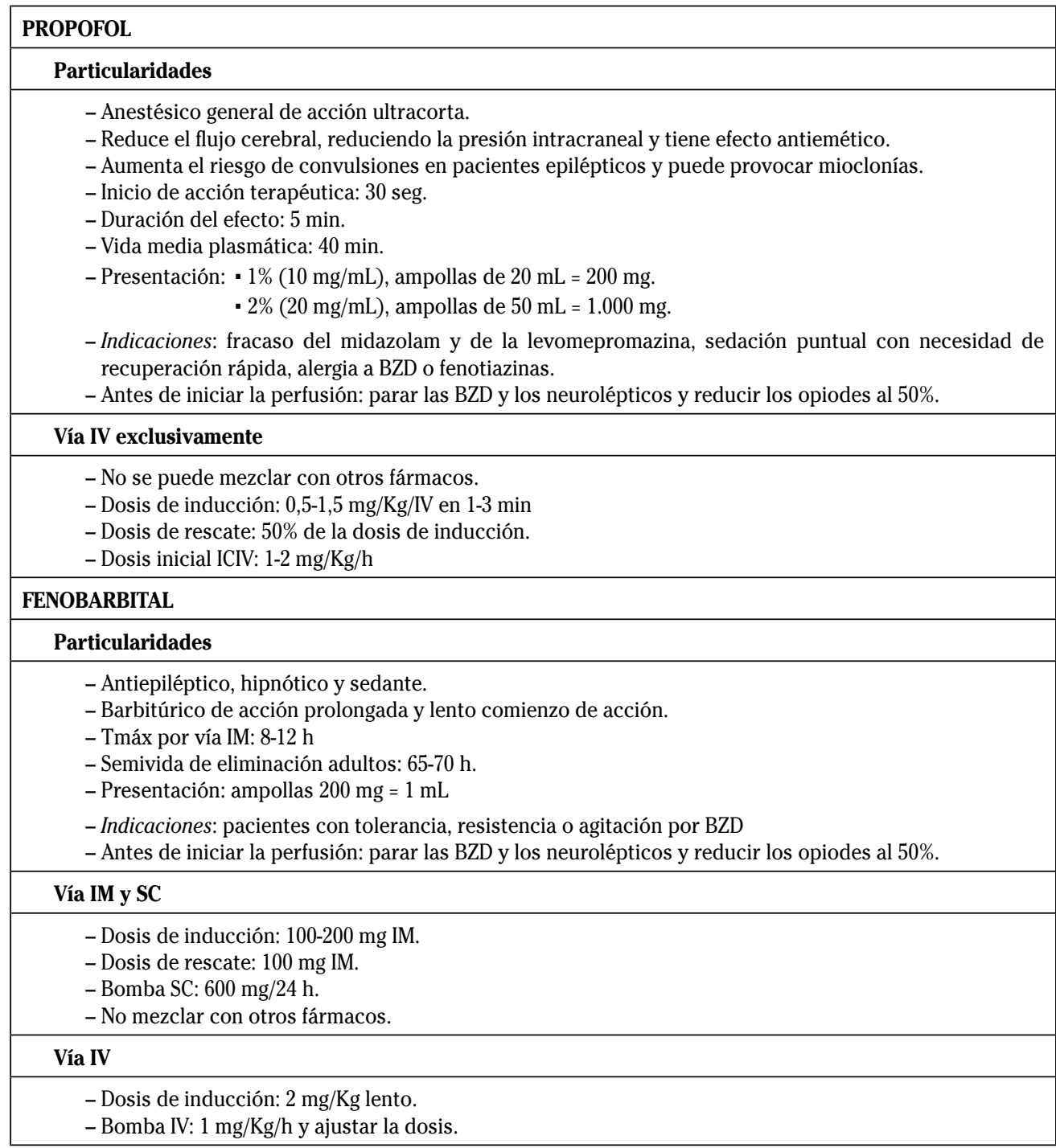

\section{CONCLUSIONES}

La gran seducción que producen los hospitales a los pacientes en SUD está en relación con la inmediatez de la respuesta, el acceso a cualquier hora y la confianza y el amparo que ofrecen por la presencia continua de los profesionales sanitarios ${ }^{6-13}$.

El alto índice de pacientes en SUD que están ubicados en los SUH está en relación con la imposibilidad del drenaje a las plan- tas de hospitalización por falta de camas hospitalarias, lo que contribuye a la mayor saturación SUH y a la inadecuada ubicación de los pacientes en SUD ${ }^{2-9}$.

Para evitar el tránsito de los pacientes en SUD por el SUH se deberá instaurar un plan de actuación específico ${ }^{10-11}$ con un soporte domiciliario que abarque desde la terapia del control de los síntomas hasta la preparación ante la muerte con un apoyo familiar progresivo a lo largo de la 
trayectoria de la enfermedad (cuidadores informados y educados sanitariamente que acepten el pronóstico), así como circuitos alternativos con acceso directo desde el propio domicilio a unidades de cuidados paliativos $^{11,14-15}$.

Si a pesar de implementar los cambios sociales y organizativos por parte de los responsables de la política sanitaria, los pacientes en SUD vienen a fallecer a urgencias, la adaptación arquitectónica de los espacios del SUH y la formación de los profesionales en estrategias y habilidades en el tratamiento de cuidados paliativos así como en decisiones morales ético-legales y en comunicación, permitirá la dignificación de un proceso habitual en la vida cotidiana: la muerte $\mathrm{e}^{6-14,18-19,53}$.

\section{BIBLIOGRAFÍA}

1. LóPEz JB. Envejecimiento y Medicina Intensiva. Med Intensiva 2005; 29: 469-474.

2. BAYÉs R. La sociedad contemporánea ante el dolor y la muerte. En: Tomás-Valiente Lanuza C. Monografía sobre la Eutanasia. Humanitas, Humanidades Médicas 2003; 1: 53-60.

3. Moreno E. ¿Y si adaptáramos los servicios hospitalarios de urgencias a la demanda social y no a las necesidades de salud? Emergencias 2008; 20: 276-284.

4. Sánchez M, Salgado E, Miró O. Mecanismos organizativos de adaptación y supervivencia de los servicios de urgencias. Emergencias 2008; 20: 48-53.

5. Miró O, Salgado E, Bragulat E, Ortega M, SalmeRón JM, SÁnchez M. Repercusión de la falta de camas de hospitalización en la actividad de un servicio de urgencias hospitalario. Med Clin (Barc) 2006; 126; 736-739.

6. Haegy JM, Andronikof M, Thiel MJ, Simon J, BiChet-Beunaiche M, Bouvier AM et al. Ethique et urgentes: réflexions de la Société Francophone de Médicine d’Urgence. Journal Europeen des Urgentes. http://www.sfmu.org (2009).

7. Iglesias ML, Villar J, Hernández E, Pallas O, GuTiÉRrez J, SkAF E. Idoneidad del proceso de fallecimiento de los pacientes que ingresan por un servicio de urgencias hospitalario. XVII Congreso Nacional de la Sociedad Española de Medicina de Urgencias y Emergencias (Palma de Mallorca). Emergencias 2005; 17: 211 .
8. Miró O, De Dios A, Antonio MT, Sánchez M, BoRRÁs A, Millá J. Estudio de la mortalidad en un servicio de urgencias hospitalario: incidencias, causas y consecuencias. Med Clin (Barc) 1999; 112: 690-692.

9. Nieto A, Arranz F, Lana R, Torres P, Rodríguez M, JimÉnEz DE Diego L. Análisis descriptivo de la mortalidad en un servicio de urgencias terciario. Emergencias 2000; 12: 291-292.

10. Mushtaq F, Ritchie D. Do we know what people die of in the emergency department? Emerg Med J 2005; 22: 718-721.

11. CHAN GK. End-of-life models and emergency departament care. Acad Emerg Med 2004; 11 : 79-86. www.aemj.org (2009).

12. Rodríguez O, Llorente S, Casanueva M, Álvarez B, Menéndez P, De la Riva G. Mortalidad en un Servicio de Urgencias Hospitalario. Características clínico epidemiológicas. Emergencias 2004; 16: 17-22.

13. RuIz V, García RF, GaGo M. Morir en Urgencias. Revista electrónica Tempus Vitalis. I Jornada de la Antigua Sociedad Española de Tanatología (SEIT) 2001. http://tanatologia.org/seit/ jornadas2001.html\#comunicaciones

14. Gómez-Batiste X, Porta-Sales J, Borrás JM, CasTELlSAgú́ X. Resource consumption and cost of palliative care services in Spain: A multicenter prospective study. J Pain Symptom Manage 2005; 31: 522-532.

15. Monés J, Forcada JM, Craven-Bartle J. Comissió de deontologia. Col-legi Oficial de Metges de Barcelona. El pols de l'ètica i la deontologia. reflexions sobre l'atenció de pacients terminals en situació d’agonia. Dossiers d'Urgències 2005; 85: 2486-2491.

16. IAE. Departament d'Estadistica. Ajuntament de Barcelona. http://www.bcn.es/estadistica (2009).

17. Comité Europeo de Salud Pública http:// www.medicosescritores.com.ar/docs/ghigi (2009).

18. Sociedad Española de Cuidados Paliativos. Guía de Cuidados Paliativos. www.seepal. com (15/10/09).

19. Porta J, Gómez-Batiste X, Tuca A. Control de síntomas en pacientes con cáncer avanzado y terminal. En: Porta J. Sedación Paliativa eds. Ed. Aran SL. Madrid, 2004.

20. Higginson IJ, Addington-Hall JM. The epidemiology of death and symptoms. En: Doyle, Hanks, Cherny and Calman. Oxford Textbook of Palliative Medicine. Ed. Oxford University Press 2004; 14-24. 
21. Rifá J, Pons O, Manzano H. El final de la vida. Síntomas y complicaciones más frecuentes. En: Manual SEDM de Cuidados Continuos 2003; 517-532.

22. Gómez-Batiste $\mathrm{X}$, Madrid J, Trelis J. Guia d’actuació en la situació d’agonia del malalt terminal. Quaderns de la bona praxis 2000; 12: 1-19.

23. Centeno C, Sanz A, Bruera E. Delirium in advanced cancer patients. Palliat Med 2004; 18 : 184-194.

24. Porta J. Aspectos éticos de la sedación en cuidados paliativos. En: Ética y sedación al final de la vida. Cuadernos de la Fundación Victor Grifols i Lucas, 2003; 9: 9-28. www. secpal.com/medicina_paliativa/index.php? (acc. 23/10/09).

25. Couceiro A. En: Ética y sedación al final de la vida. Cuadernos de la Fundación Victor Grifols i Lucas, 2003; 9: 29-58. www.secpal. com/medicina_paliativa/index.php? (acc. 23/10/09

26. Morita T. Palliative sedation to relieve psycho-existential suffering of terminally-ill cancer patients. J Pain Symptom Manage 2004; 28: 445-450.

27. GonzÁlez M, Gómez C, Vilchez Y. Última etapa de la enfermedad neoplásica progresiva: cuidados en la agonía, síntomas refractarios y sedación. Med Clin (Barc) 2006: 127: 421-428.

28. Rousseau P. Palliative sedation in the control of refractory symptoms. J Palliat Med 2005; 8: 10-12.

29. TuCA A. Tratamiento de síntomas refractarios. Sedación, sus indicaciones y la ética. En: Manual SEDM de Cuidados Continuos 2003; 533-553.

30. SAnz J. End-of-life sedation. Med Clin (Barc) 2004; 123: 423-425.

31. GARcía ED. Cuidados en la situación de agonía. En: Valentín V, Alonso C, Murillo MT, Pérez $\mathrm{P}$, Vilches V eds. Nova Sidonia Oncología y Hematología. Madrid 2002; 653-662.

32. Des Plaines IL. End-of-life care in the emergency Department. Emergency Nurses Association Position, 2001.

33. Arteta A. La buena muerte. En: Astudillo W, Morales A, Clavé E, Cabarcos A y Urdaneta E eds. Avances recientes en Cuidados Paliativos. Sociedad Vasca de Cuidados Paliativos 2002; 297-311.

34. Bassols A, Cañellas M, Bosch F, Baños JE. Per què encara es controla tan malament el dolor?: Dades, reflexions i propostes de millota: Annals de Medicina 2005; 88: 4-6.
35. GRAcia D, Júdez J (eds). Ética en la práctica clínica. Ed. Triacastela, Madrid 2004: 21-32.

36. Beauchamp TL, Childress JF. Principles of Biomedical Ethics. $4^{\mathrm{a}}$ ed. New York: Oxford University Press, 1994.

37. Iglesias ML, Pedro-Botet JC, Gutiérrez J, HernÁndez E, Pallás O, Aguirre A et al. Análisis ético de las decisiones médicas en el servicio de urgencias de un hospital universitario. Emergencias 2000; 12: 313-322.

38. Armengol R, Boladeras M, Broggi MA, Camps V, Espasa R, Hernández J et al. Comitè Consultiu de Bioètica de Calalunya. Informe sobre la eutanasia y la ayuda al suicidio. Generalitat de Catalunya. Departament de Salut.

39. Herranz G. Eutanasia y dignidad de morir. Jornadas Internacionales de Bioética, Bioética y dignidad en una sociedad plural. http:// www.bioeticaweb.com (5/10/2009).

40. Cabre L, Solsona JF. Limitación del esfuerzo terapéutico en medicina intensiva. Med Intensiva 2002; 26: 304-311.

41. Fernández R, Baigorri F, Artigas A. Limitación del esfuerzo terapéutico en Cuidados Intensivos ¿Ha cambiado en el siglo XXI? Med Intensiva 2005; 29: 338-341.

42. Simón P, Barrio IM, Alarcos FJ, Barbero J, CouCEIRo A, Hernando P. Ética y muerte digna: propuesta de consenso sobre un uso correcto de palabras. Rev Calidad Asistencial 2008; 23: 271-285.

43. CABRÉ LL. Síndrome de fracaso multiorgánico y limitación al esfuerzo terapéutico. REMI 2004; 4: C33. http://remi.uninet.edu (2009).

44. Núñez S, Marco T, Burillo-Putze G, Ojeda J. Procedimientos y habilidades para la comunicación de las malas noticias en urgencias. Med Clin (Barc) 2006; 127: 580-583.

45. Astudillo W, Mendinueta C, Astudillo E. Cuidados del enfermo en fase terminal y atención a su familia. EUNSA, Barañain, 4⿳亠丷厂 Edición, 2002: 227-246.

46. TucA A. Tratamiento de síntomas refractarios. Sedación, sus indicaciones y la ética. En: Campos C, Carulla J, Casas AM, González M, Sanz-Ortíz J, Valentín V eds. Manual SEOM de cuidados continuos. Ed. Dispublic SL Madrid, 2004.

47. Brown JE, Hamilton Glenn. Malas noticias: notificación de defunción. En: Tintinalli JE, Ruiz E, Krome RL, editores. Medicina de Urgencias. American College of Emergency Physicians. McGraw-Hill, Nueva York 2005; 22: 4-7.

48. Ayarra M, Lizarraga S. Malas noticias y apoyo emocional. An Sist Sanit Navar 2001 (Supl. 2): 55-63. 
49. ElLERSHAW J, WARD C. Care of the dying patient: the last hours or days of life. BMJ 2003; 326: 30-34.

50. Arteta A. La buena muerte. En: Astudillo W, Morales A, Clavé E, Cabarcos A y Urdaneta E eds. Avances recientes en Cuidados Paliativos. Sociedad Vasca de Cuidados Paliativos 2002; 297-311.

51. Montes-Romero JA, Rodríguez-Galdeano M, Cervantes-Bonet B, Marín-Gámez N. About taking decisions at the the end of live. Med Clin (Barc) 2003; 121: 318.

52. Arranz P, Barbero JJ, Barreto P, Bayés R. Intervención emocional en cuidados paliativos. Modelo y protocolos. Ariel SA. Barcelona, 2003

53. De Miguel C, López A. El paciente con cáncer avanzado. ¿Podemos predecir la supervivencia? Aten Primaria 2006; 38 (Supl. 2): 2-6. htpp://www.doyma.es (16/10/2009).

54. Puchalski C. Spirituality in health: the role of spirituality in critical care. Crit Care Clin 2004; 20: 487-504.

55. McClain-Jacobson C, Rosenfeld B, Kosinski A, Pessin H, Cimino JE, Breitbart W. Belief in an afterlife, spiritual wellbeing and end-of-life despair in patients with advanced cancer. Gen Hosp Psychiatry 2004; 26: 484-486.
56. BiJur PE, Kenny MK, Gallagher EJ. Intravenous morphine at $0.1 \mathrm{mg} / \mathrm{kg}$ is not effective for controlling severe acute pain in the majority of patients. Ann Emerg Med 205; 46: 362-367.

57. Birnbaum A, Esses D, BiJur PE, Holden L, GallaGHER EJ. Randomized doble-blind placebo-controlled trial of two intravenous morphine dosages $(0.10 \mathrm{mg} / \mathrm{kg}$ and $0.15 \mathrm{mg} / \mathrm{kg})$ in emergency department patients with moderate to severe acute pain. Ann Emerg Med 2007; 49: 445-453.

58. Aubrun F, Salvi N, Coriat P, Riou B. Sex-and age-related differences in morphine requirements for postoperative pain relief. Anesthesiology 2005; 105: 156-160.

59. Goenaga MA, Millet M, Carrera JA, Garde C. Vía subcutánea: más fármacos. Med Pal 2004; 7: 28. www.secpal.com/medicina_paliativa/index.php? (acc. 23/10/09).

60. Ahmedzai S. Palliation of respiratory symptoms. En: Doyle D, Hanks GW, Mac Donald N. Oxford Textbook of palliative medicine $2^{\mathrm{a}} \mathrm{ed}$. Oxford University Press, 1998; 583-616.

61. Navigante AH, Cerchietti LC, Castro MA, LutteRAL MA, CABALAR ME. Midazolam as adjunct therapy to morphine in the alleviation of severe dyspnea perception in patients with advanced cancer. J Pain Symptom Manage 2006; 31: 38-47. 
